\title{
Mind Controlled Drone: An Innovative Multiclass SSVEP based Brain Computer Interface
}

Chiuzbaian, Andrei; Jakobsen, Jakob; Puthusserypady, Sadasivan

\section{Published in:}

Proceedings of 2019 7th International Winter Conference on Brain-Computer Interface

Link to article, DOI:

10.1109/IWW-BCI.2019.8737327

Publication date:

2019

Document Version

Peer reviewed version

Link back to DTU Orbit

\section{Citation (APA):}

Chiuzbaian, A., Jakobsen, J., \& Puthusserypady, S. (2019). Mind Controlled Drone: An Innovative Multiclass SSVEP based Brain Computer Interface. In Proceedings of 2019 7th International Winter Conference on BrainComputer Interface IEEE. https://doi.org/10.1109/IWW-BCI.2019.8737327

\section{General rights}

Copyright and moral rights for the publications made accessible in the public portal are retained by the authors and/or other copyright owners and it is a condition of accessing publications that users recognise and abide by the legal requirements associated with these rights.

- Users may download and print one copy of any publication from the public portal for the purpose of private study or research.

- You may not further distribute the material or use it for any profit-making activity or commercial gain

- You may freely distribute the URL identifying the publication in the public portal 


\section{Mind Controlled Drone: An Innovative Multiclass SSVEP based Brain Computer Interface}

\author{
Andrei Chiuzbaian \\ Dept. of Electrical Engineering \\ Technical University of Denmark \\ Lyngby, Denmark \\ andrei.chiuzbaian@gmail.com
}

\author{
Jakob Jakobsen \\ Dept. of Space Research \& Space Technology \\ Technical University of Denmark \\ Lyngby, Denmark \\ jj@space.dtu.dk
}

\author{
Sadasivan Puthusserypady \\ Dept. of Electrical Engineering \\ Technical University of Denmark \\ Lyngby, Denmark \\ spu@elektro.dtu.dk
}

\begin{abstract}
A crucial element lost in the context of a neurodegenerative disease is the possibility to freely explore and interact with the world around us. The work presented in this paper is focused on developing a brain-controlled Assistive Device (AD) to aid individuals in exploring the world around them with the help of a computer and their thoughts. By using the potential of a noninvasive Steady-State Visual Evoked Potential (SSVEP)based Brain Computer Interface (BCI) system, the users can control a flying robot (also known as UAV or drone) in 3D physical space. From a video stream received from a video camera mounted on the drone, users can experience a degree of freedom while controlling the drone in 3D. The system proposed in this study uses a consumer-oriented headset, known as Emotiv Epoch in order to record the electroencephalogram (EEG) data. The system was tested on ten able-bodied subjects where four distinctive SSVEPs $(5.3 \mathrm{~Hz}, 7 \mathrm{~Hz}, 9.4 \mathrm{~Hz}$ and $13.5 \mathrm{~Hz}$ ) were detected and used as control signals for actuating the drone. A highly customizable visual interface was developed in order to elicit each SSVEP. The data recorded was filtered with an $8^{\text {th }}$ order Butterworth bandpass filter and a fast Fourier transform (FFT) spectral analysis of the signal was applied in other to detect and classify each SSVEP. The proposed BCI system resulted in an average Information Transfer Rate (ITR) of $10 \mathrm{bits} / \mathrm{min}$ and a Positive Predictive Value (PPV) of $92.5 \%$. The final conducted tests have demonstrated that the system proposed in this paper can easily control a drone in $3 D$ space.

Index Terms-Brain Computer Interface (BCI), Electroencephalogram (EEG), Steady State Visual Evoked Potential (SSVEP), Drone Control, Assistive Device (AD), Information Transfer Rate (ITR).
\end{abstract}

\section{INTRODUCTION}

"Disability should never be a barrier in one's success.” - Stephen Hawking.

Worldwide, one person out of five suffers from some form of disability according to the 2011 WHOs world report on disability [1]. This global estimation is on the rise due to aging population and a rapid spread of chronic diseases. Many of these people suffer from neuromuscular disorders such as amyotrophic lateral sclerosis (ALS), spinal cord injury, brainstem stroke and many other disorders responsible for causing the loss of voluntary muscle control. Such people are often locked in a wheelchair or on a bed unable to move their limbs or go anywhere they would like to go by themselves. They have to face great barriers in the modern society due to their disabilities and deprivation of common activities like interacting or playing games with other people, activities that are crucial for personal development and have a significant impact on the quality of life. Those with a lack of motor skills would benefit enormously from devices that can augment their mobility. Over the last few years, the state of the art technology known as the Brain-Computer Interface (BCI) has become more and more accessible to the wider public and it is our moral responsibility to use such technologies in order to lift these barriers and give disabled people a chance to regain a normal life. There has been a lot of work in this direction during the past few years where researchers have tried innovative solutions for developing a user-friendly and easy to use assistive systems for controlling a drone. In 2012, Yipeng et al designed a BCI system that was using motor imagery (MI) signals acquired from thinking left, thinking right and thinking push combined with the artifact signals from eye blinking and tooth clenching in order to control an AR drone [2]. A different setup was suggested by Byung et al, where a hybrid interface was used [3]. In their study, the drone was controlled by using a low-cost electroencephalographic (EEG) headset together with an eye-tracking device. Although the BCI systems presented by previous authors come as affordable solutions for those who want to control a drone with their minds, the same studies have confirmed that BCI systems based on motor imagery commands are susceptible to artifacts like inappropriate eye blinking or muscle activity.

The novelty of the work presented in this study is in achieving a user-friendly, fully independent multi-class BCI system based on the Steady State Visual Evoked Potential (SSVEP) paradigm that allow users to control a drone in 3D physical space only by using their EEG signals. In addition, the system we propose is ready-to-go which means users do not require any previous training or experience in order to actuate the drone.

\section{System ARChitecture AND IMPLEMENTATION}

The BCI system architecture can be seen in Fig.1. The main components of the system are: a custom-made visual interface, EEG headset, a laptop used for signal acquisition and processing, and a wireless drone. 


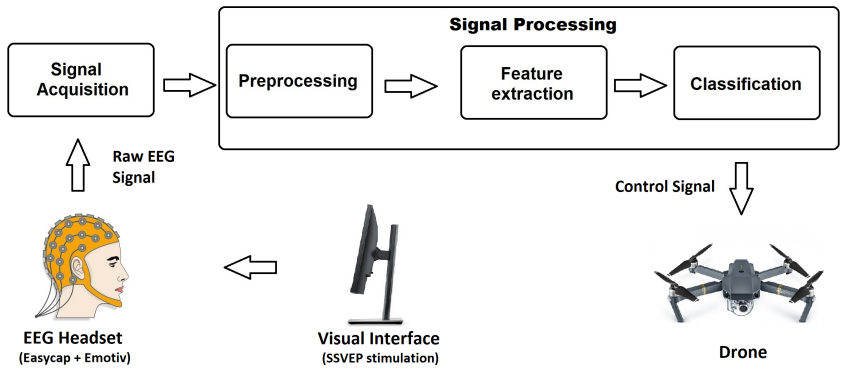

Fig. 1. The proposed BCI system architecture.

\section{A. Visual Interface}

The BCI system developed in this paper is based on a SSVEP paradigm. In order to stimulate the SSVEPs, a visual interface (Fig.2) was designed. The interface was developed in C\# and implemented in Visual Studio 2017. The application runs on a 24-inch computer screen with a refresh rate of 120 $\mathrm{Hz}$. It consists of four arrow-like stimuli placed equidistant at the top, bottom, left and right sides of the screen. Each stimulus represents a movement command that actuates the drone in a specific direction. A high contrast color (black/white) scheme was chosen for our application and plain textures for the visual stimuli in order to increase the chances of evoking SSVEPs [4] [5] [6]. Furthermore, the luminance contrast level was increased by adjusting the contrast and brightness levels on the digital display [7].

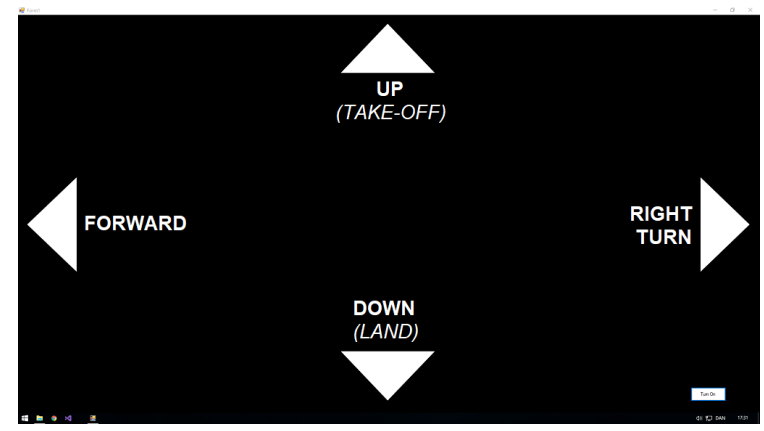

Fig. 2. The visual interface used for evoking SSVEPs.

The stimulation frequencies $\left(f_{\mathrm{U}}, f_{\mathrm{D}}, f_{\mathrm{L}}\right.$, and $\left.f_{\mathrm{R}}\right)$ for evoking each SSVEP together with the corresponding movement command for the drone can be seen in Table I. A combination of turn right and then go forward commands was used in order to compensate for the lack of left-right strafe movements.

TABLE I

THE DRONE CONTROL COMMANDS AND THEIR CORRESPONDING STIMULATION FREQUENCIES.

\begin{tabular}{c|c|c}
\hline Arrow & Stimulus frequency [Hz] & Command \\
\hline \hline Up & $f_{\mathrm{U}}$ & Take-off \\
Down & $f_{\mathrm{D}}$ & Land \\
Left & $f_{\mathrm{L}}$ & Forward \\
Right & $f_{\mathrm{R}}$ & Right turn \\
\hline \hline
\end{tabular}

\section{B. EEG Headset}

For the BCI system proposed in this paper, a consumeroriented EEG headset (shown in Fig.3) was used as it is non-invasive, affordable and most important, portable. The solution was to use the hardware unit from an Emotiv EPOC neuroheadset connected to the EEG-recording cap made by Easycap.

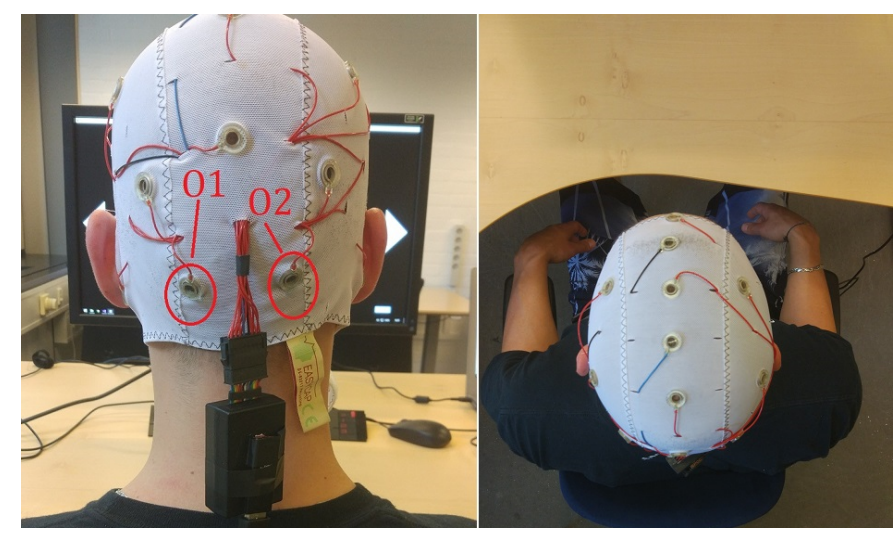

Fig. 3. The EEG headset with the two main electrodes used for recording the encephalic activity.

The cap comes equipped with 14 recording electrodes and two reference electrodes (CMS and DRL). All 16 electrodes are $\mathrm{AgCl}$ electrodes able to provide superior EEG recording quality over the gold-plated electrodes in the Emotiv EPOC's original design. The EEG headset is used to extract the encephalic activity from the visual cortex in the posterior pole of the occipital lobe. The raw EEG signals recorded from channels $\mathrm{O} 1$ and $\mathrm{O} 2$ are sampled by the Emotiv EPOC's hardware with a sampling rate of $128 \mathrm{~Hz}$ and a $50 \mathrm{~Hz}$ digital notch filter is applied over the data in order to remove any EM interference emitted by the power lines. The data collected by the EEG headset is sent via Bluetooth to a laptop with a USB dongle.

\section{Signal Processing}

In order to process the EEG signals, several software tools were used. For signal acquisition and for decryption of the raw EEG data, a script was developed and implemented in Python. The signal processing, feature extraction, and classification algorithms were performed in Matlab. In order to send the EEG data from Python to Matlab, a software tool known as Lab Streaming Layer (LSL) was used. The analysis of the EEG data is performed in real time over a succession of time lapses. The time lapses were divided into two-second intervals and processed individually. The data was filtered afterwards by using an $8^{\text {th }}$ order Butterworth bandpass $(2-15 \mathrm{~Hz})$ filter and normalized by using zero-mean and unit variance techniques. A fast Fourier transform (FFT) algorithm was used for the feature detection of the SSVEPs. For classifying each SSVEP, a threshold technique was used and specific threshold levels were applied on both frequency and magnitude axis. If the peak value of one of the SSVEPs is evoked within its 
specific detection area during the execution of the program, the program will generate an output command. The generated output command will be translated into one of the four control commands that will be used for flying the drone. The FFT spectral analysis performed on the EEG data is illustrated in Fig.4.

\section{FFT Spectral Analysis}

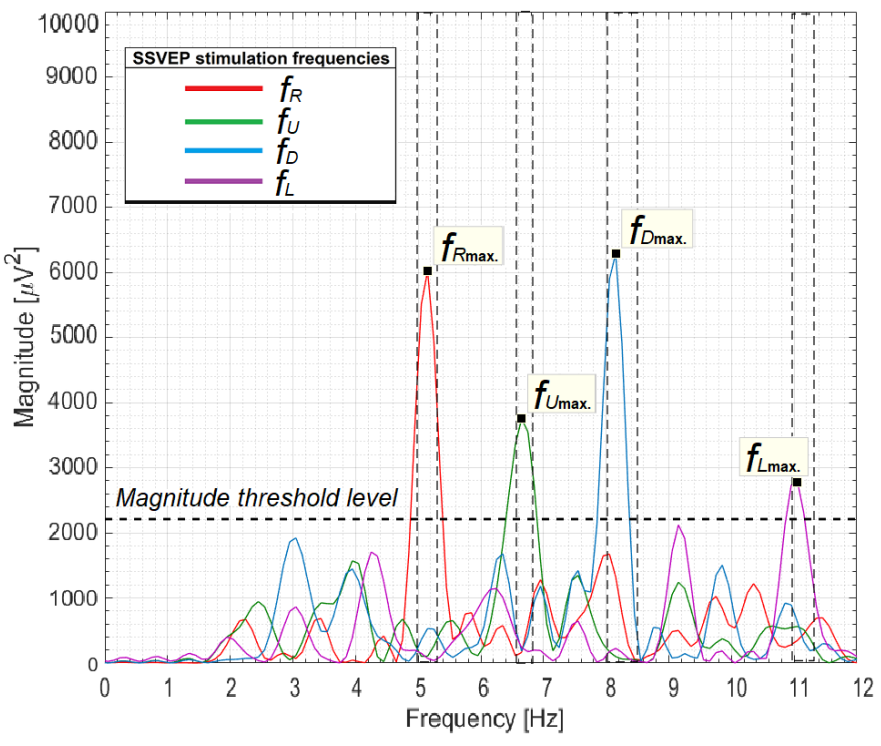

Fig. 4. The frequency and magnitude levels of each SSVEP and their detection.

In this FFT spectral analysis, each of the four SSVEPs is marked with a different color and their maximum frequency and magnitude values are displayed in a text box next to their maxima point. The frequency threshold levels for the minimum $\left(F_{\text {min. }}\right)$ and maximum $\left(F_{\text {max }}\right)$ values for each SSVEP is marked with a vertical dashed line and the magnitude threshold level is marked with a horizontal dashed line. The $F_{\text {min. }}$ and $F_{\text {max }}$. threshold values for each SSVEP can be seen in Table II. From the tests performed during the project, we noticed that the background noise in the EEG signals was peaking at about $2000 \mu \mathrm{V}^{2}$ on the magnitude axis and therefore we set the max. threshold value to $2200 \mu \mathrm{V}^{2}$ to avoid any false SSVEP detection.

TABLE II

THE FREQUENCY AND MAGNITUDE THRESHOLD VALUES SET FOR EACH SSVEP.

\begin{tabular}{c|c|c|c}
\hline SSVEP $[\mathrm{Hz}]$ & $F_{\text {min. }}[\mathrm{Hz}]$ & $F_{\max .}[\mathrm{Hz}]$ & Magnitude $\left[\mu \mathrm{V}^{2}\right]$ \\
\hline \hline$f_{\mathrm{R}}$ & 5.0 & 5.3 & 2200 \\
$f_{\mathrm{U}}$ & 6.6 & 6.8 & 2200 \\
$f_{\mathrm{D}}$ & 8.0 & 8.5 & 2200 \\
$f_{\mathrm{L}}$ & 10.9 & 11.3 & 2200 \\
\hline \hline
\end{tabular}

\section{EXPERIMENTAL PROTOCOL}

All the experiments performed during this project were conducted at the Technical University of Denmark in the
Biomedical Engineering Department. The provided room was partly phonically isolated. The experiments were performed during daytime in a fully lighted room. In total, two studies were carried out. The first study consisted of a set of experiments performed on three volunteers in order to test different SSVEP responses. In the second study, a total of ten volunteers participated in the tests in order to evaluate the performance of the real-time BCI system. All participants in the experiments were healthy individuals with normal or corrected to normal vision (wearing contact lenses), and none of them suffered from epilepsy. All subjects were provided verbal informed consent prior to their participation in the experiments, which were approved by the Regional Committee on Health Research Ethics for the Capital Region of Denmark (reference H-3-2013-004) and carried out in accordance with the corresponding guidelines and relevant regulations on the use of human subjects for health-related scientific research.

Each individual was seated comfortably in an armchair with their forearms placed flat at $90^{\circ}$ on the armrests and their feet resting flat on the ground. During the recordings, the computer screen running the visual interface was placed at approximately $1 \mathrm{~m}$ above the ground and $0.6 \mathrm{~m}$ from the subject's eyes. The EEG cap was placed on the subject's head and secured with a velcro strap across the chin. A highly conductive electrolyte gel had to be applied in between the subject skin and the electrodes in order to increase the signal conductivity. As the experiments had to be conducted indoor, due to safety reasons, the drone had to be replaced with a feedback circuit. The feedback circuit consisted of four LEDs controlled by an Arduino board. Each LED was turned on depending on the SSVEP detected by the BCI system. For example, when the subject was gazing at the left arrow on the screen the corresponding $13.5 \mathrm{~Hz}$ SSVEP was elicited and converted into a control signal that turned on the left LED. The experimental setup and the LED circuit are illustrated in Fig.5.
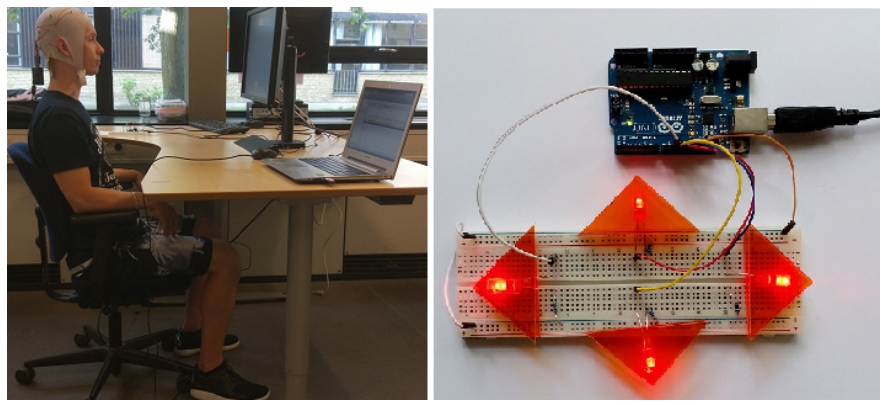

Fig. 5. The experimental setup (left) and the LED circuit (right).

\section{RESUlts}

In order to assess the performance of the BCI system proposed in this paper, a scenario was created where each subject had to control the drone on a predefined path. The command sequence used during the test trials can be seen in Table III. 
The subjects were instructed to gaze at one stimulus at a time. Once the detection of the SSVEP occurred, the subject had to change their gaze to the next stimulus in the sequence. According to the feedback received from the BCI system the commands were classified as Correct $(C)$, Incorrect $(I)$ or Not Detected $(N D)$. For evaluating the performance of the BCI system, the Information Transfer Rate $(I T R)$ was used as a measure. The ITR was calculated (Eq.(1)) as suggested in [8].

$$
I T R=p_{\mu}^{\prime}\left[\log _{2} N+p_{e}^{\prime} \log _{2} p_{e}^{\prime}+p_{e} \log _{2}\left(\frac{p_{e}}{N-1}\right)\right],
$$

where $N$ is the number of stimuli (in this case $N=4$ ), $p_{\mu}^{\prime}=1-$ $p_{\mu}$, and $p_{e}^{\prime}=1-p_{e} \cdot p_{\mu}$ and $p_{\mathrm{e}}$ here represents the probability of undefined cases and the probability of incorrect detected cases, respectively and are defined as:

$$
\begin{aligned}
p_{\mu} & =\frac{N D}{C+I+N D}, \\
p_{e} & =\frac{I}{C+I+N D} .
\end{aligned}
$$

The ITR is expressed in bits/command. In order to obtain the result in bits/min, the value had to be multiplied with the detection speed of the BCI system which was 20 commands/min. The ITR achieved by each subject during the evaluation process of the BCI system can be seen in Fig. 6.

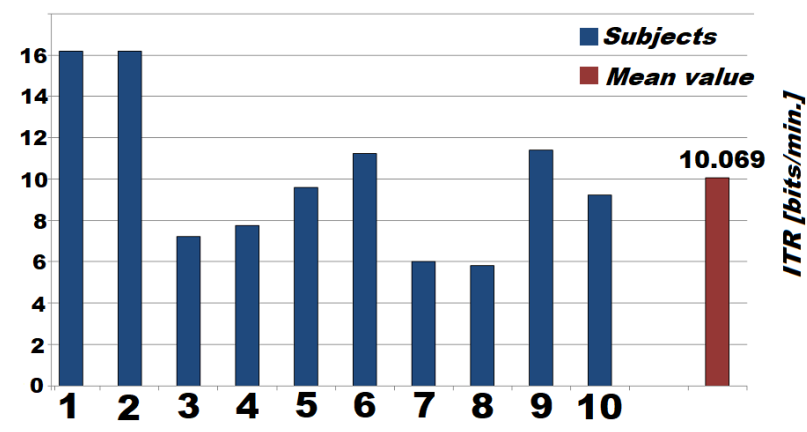

Fig. 6. The ITR achieved by each subject and the mean value achieved by the BCI system.

Another metric used for evaluating the performance of the BCI system is the Positive Predictive Value (PPV). It

TABLE III

THE STIMULI SEQUENCE USED DURING THE EVALUATION PROCESS OF THE BCI SYSTEM.

\begin{tabular}{c|c|c}
\hline Sequence & Command & Stimulus \\
\hline \hline 1 & Take off & UP \\
2 & Forward & LEFT \\
3 & Turn right & RIGHT \\
4 & Decrease altitude & DOWN \\
5 & Forward & LEFT \\
6 & Increase altitude & UP \\
7 & Turn right & UP \\
8 & Land & DOWN \\
\hline \hline
\end{tabular}

represents the proportion of SSVEPs that are correctly detected and it can be obtained by applying the following formula:

$$
P P V=\frac{T P}{T P+F P},
$$

where TP stands for all the true positive commands and FP for all the false positive commands send by the BCI system to the drone when an SSVEP was detected. A TP command represents a successfull detection of an SSVEP by the BCI system while the FP command is an erroneous signal interpreted by the BCI system as an SSVEP.

In this study, the BCI system achieved a mean PPV of $92.5 \pm 8.29 \%$ across ten subjects. The results obtained form the subjects who participated in the evaluation stage of the BCI system is illustrated in Table IV. The table presents the elapsed time, the number of output commands sent by the BCI system to the drone, and the ITR and PPV achieved by each subject. As can be seen from the table, 5 out of 10 subjects achieved a PPV of $100 \%$.

TABLE IV

THE RESULTS OBTAINED BY THE SUBJECTS DURING THE EVALUATION PROCESS OF THE BCI SYSTEM.

\begin{tabular}{c|c|c|c|c|c|c}
\hline \multirow{2}{*}{ Subject } & Time & \multicolumn{3}{|c|}{ Commands } & ITR & PPV \\
\cline { 3 - 5 } & $(\mathrm{s})$ & $\mathbf{C}$ & I & ND & (bits/min.) & $(\%)$ \\
\hline \hline 1 & 62 & 8 & 0 & 22 & 16.20 & 100.0 \\
2 & 60 & 8 & 0 & 22 & 16.20 & 100.0 \\
3 & 124 & 7 & 1 & 54 & 7.23 & 87.5 \\
4 & 118 & 7 & 1 & 51 & 7.76 & 87.5 \\
5 & 100 & 8 & 0 & 42 & 9.60 & 100.0 \\
6 & 78 & 7 & 1 & 31 & 11.24 & 87.5 \\
7 & 156 & 8 & 0 & 70 & 6.00 & 100.0 \\
8 & 140 & 6 & 2 & 62 & 5.82 & 75.0 \\
9 & 84 & 8 & 0 & 34 & 11.40 & 100.0 \\
10 & 92 & 7 & 1 & 38 & 9.24 & 87.5 \\
\hline Mean & $\mathbf{1 0 1}$ & $\mathbf{7 . 4}$ & $\mathbf{0 . 6}$ & $\mathbf{4 2 . 6}$ & $\mathbf{1 0 . 0 6 9}$ & $\mathbf{9 2 . 5}$ \\
\hline Std. dev. & $\mathbf{3 0 . 7 6}$ & $\mathbf{0 . 6 6}$ & $\mathbf{0 . 6 6}$ & $\mathbf{1 5 . 5 1}$ & $\mathbf{3 . 5 7}$ & $\mathbf{8 . 2 9}$ \\
\hline \hline
\end{tabular}

\section{Discussion}

The results from the study presented in this paper have demonstrated that people are able to control a drone in 3D space with the state of the art technology, known as the BCI [9] [10] [11] [12]. By presenting visual stimuli in front of user's retina, evoked brain signals (SSVEP) can be recorded from the occipital region of the brain using EEG techniques [13] [14] [15] [16]. Through signal processing, these signals can be translated into control commands for actuating different electronic devices such as wheelchairs [17] [18] [19] [20], robotic arms [21] [22] or, like in our study, a drone [23]. The BCI system presented in this paper is based on a four-class SSVEP model. In order to improve the control scheme of the drone in 3D, the system can be extended to a six-class SSVEP model in which six distinct SSVEP will be used to actuate the drone. The BCI system presented in this work has achieved a moderate ITR of $10 \mathrm{bits} / \mathrm{min}$. which means the system will be able to output in average 10 control commands every minute, making the whole system relatively slow in comparison with 
controlling the drone from a remote controller. For future improvements, the authors recommend the use of a premium EEG device like a clinical or research-oriented neuroheadset. It is worth mentioning that the experiments done during this project have been conducted in a fully-lighted room during daytime which may have affected the quality of the SSVEP detection. The authors would recommend that the future work done on SSVEP-based BCIs to be performed in dark rooms which can result in a better SSVEP detection.

\section{CONCLUSION}

The goal of this work was to develop a user-friendly, fullyindependent BCI system that will allow impaired people to control a drone in 3D space by using only their brain. The application intends to enhance the quality of life allowing users to interact with the environment around them. The system is based on a 4-class SSVEP model able to move the drone up/down, left/right, forward/backward. The BCI system was tested on ten able-bodied control subjects and by using standard metrics it was able to achieve an average accuracy of $92.5 \%$ and an average ITR of $10 \mathrm{bits} / \mathrm{min}$. A video with the BCI system controlling the drone in real-time can be found on the following link [23].

\section{ACKNOWLEDGEMENTS}

The authors would like to thank the support from DTU Space for allowing us to access to the drone which was developed in their laboratory. The EEG equipment used in this study was provided by the Department of Computer Engineering and Department of Electrical Engineering at DTU. We acknowledge these departments for their support. The authors would like to thank all the volunteers who participated in the experiments and give special thanks to all the colleagues in the Bio-medical Engineering group (DTU) who contributed with insightful ideas and feedback.

\section{REFERENCES}

[1] J. R.Wolpaw, N. Birbaumer, D. J. McFarland, G. Pfurtscheller, and T. M. Vaughan, "Brain-computer interfaces for communication and control", Clinical Neurophysiology, Vol. 113, No.6, pp. 767-791, June 2002.

[2] Y. Yu, D. He, W. Hua, S. Li, Y. Qi, Y. Wang, and G. Pan, "Flyingbuddy2: A brain-controlled assistant for the handicapped", In Proceedings of the 2012 ACM Conference on Ubiquitous Computing, Pittsburgh, PA, USA, pp. 669-670, 05-08 September, 2012.

[3] J.-S. Lin, and Z. Y. Jiang, "Implementing remote presence using quadcopter control by a non-invasive BCI device", Computer Science and Information Technology, ISSN 1820-0214, Vol. 3, No.4, pp. 122-126, May 2015.

[4] J. Bieger, G. G.-Molina, and D. Zhu, "Effects of stimulation properties in steady-state visual evoked potential based brain-computer interfaces", In Proceedings of the 34th IEEE International Conference of IEEE EMBS, Buenos Aires, Argentina, pp. 3345-3348, August 2010.

[5] T. Cao, F. Wan, P. U. Mak, P.-I. Mak, M. I. Vai, and Y. Hu, "Flashing color on the performance of ssvep-based brain-computer interfaces", In Proceeding of Annual International Conference of the IEEE EMBS, San Diego, CA, USA, pp. 1819-1822, 28 August-1 September 2012.

[6] B. Z. Allison, I. Sugiarto, B. Graimann, and A. G. P. Graser, "Display optimization in SSVEP BCI", IEEE International Conference on Computer Engineering and Technology, at Singapore, Singapore, 22-24 January 2009.
[7] A. Duszyk, M. Bierzynska, Z. Radzikowska, P. Milanowski, R. Kus, P. Suffczynski, M. Michalska, M. Labecki, P. Zwolinski, and P. Durka, "Towards an optimization of stimulus parameters for brain-computer interfaces based on steady state visual evoked potentials", Plos One, Vol. 9, No.11, November 2014

[8] P. F. Diez, S. M. T. Muller, V. A. Mut, E. Laciar, E. Avila, T. F. B.Filho, and M. S.-Filho, "Commanding a robotic wheelchair with a highfrequency steady-state visual evoked potential based brain-computer interface", Medical Engineering Physics, Vol. 35, No.8, pp. 1155-1164, August 2013.

[9] K. LaFleur, K. Cassady, A. Doud, K. Shades, E. Rogin, and B. He, "Quadcopter control in three-dimensional space using a noninvasive motor imagery-based brain- computer interface", Journal of Neural Engineering, Vol. 10, No.4, June 2013.

[10] B.H. Kim, M. Kim, and S. Jo, "Quadcopter flight control using lowcost hybrid interface with EEG-based classification and eye tracking", Computers in Biology and Medicine, Vol. 51, pp. 82-92, doi: 10.1016/j.compbiomed.2014.04.020, August 2014.

[11] M. Al-Ayyoub, A.-H. A. A.-Rahman, Y. Qadoumi, M. B. Hani, M. Masadeh, R. A. A.-Asal, and M. B. Aboud, "Mind-Controlling Green Unmaned Vehicles through the Cloud: Building a Prototype", Journal of Advances in Informational Technology, ISSN: 1798-2340, Vol. 6, No.1, February 2015.

[12] N. Kosmyna, F. T.-Bernard and B. Rivet, "Towards Brain Computer Interfaces for Recreational Activities: Piloting a Drone", In Proceedings of the 15th International Federation for Information Processing, Part I, ISSN 0302-9743, pp. 506-522, Bamberg, Germany, September 14-18, 2015.

[13] G. R. M.-Putz, R. Scherer, C. Brauneis, and G. Pfurtscheller, "Steadystate visual evoked potential (SSVEP)-based communication: impact of harmonic frequency components", Journal of Neural Engineering, vol. 2, No.4, pp. 123-130, December 2005.

[14] K. B. Ng, A. P. Bradley, R. Cunnington, "Stimulus specificity of a steady-state visual-evoked potential-based brain-computer interface", Journal of Neural Engineering, Vol. 9(3), May 2012.

[15] Z. Wu, Y. Lai, Y. Xia, D. Wu, and D. Yao, "Stimulator selection in SSVEP-based BCI", Medical Engineering \& Physics, Vol 30, No.8, pp. 1079-1088, October 2008.

[16] B. Allison, T. Luth, D. Valbuena, A. Teymourian, I. Volosyak, and A. Graser, "BCI Demographics: How Many (and What Kinds of) People Can Use an SSVEP BCI?", IEEE Transactions on Neural Systems and Rehabilitation Engineering, vol. 18, No.2, April 2010.

[17] C. Mandel, T. Luth, T. Laue, T. Rofer, A. Graser, and B. K-Bruckner, "Navigating a Smart Wheelchair with a Brain-Computer Interface Interpreting Steady-State Visual Evoked Potentials", In Proceedings of the International Conference on Intelligent Robots and Systems, St. Louis, MO, USA, 10-15 October 2009.

[18] J. Philips, J. R. Millan, G. Vanacker, E. Lew, F. Galan, P. W. Ferrez, H. V. Brussel, and M. Nuttin, "Adaptive Shared Control of a Brain-Actuated Simulated Wheelchair", In Proceedings of the IEEE 10th International Conference on Rehabilitation Robotics, at Noordwijk, Netherlands, 1315 June 2007.

[19] S. M. T. Muller, T. F. Bastos, and M. S. Filho, "Proposal of a SSVEP-BCI to Command a Robotic Wheelchair", Journal of Control, Automation and Electrical Systems, Vol. 24, No.1-2, pp. 97-105, April 2013.

[20] M. Palankar, K. J. D. Laurentis, R. Alqasemi, E. Veras, R. Dubey, Y. Arbel, and E. Donchin, "Control of a 9-DoF Wheelchair-Mounted Robotic Arm System Using a P300 Brain Computer Interface: Initial Experiments", In Proceedings of the IEEE International Conference on Robotics and Biomimetics, at Bangkok, Thailand, 22-25 February 2009.

[21] G. R. M.-Putz, and G. Pfurtscheller, "Control of an Electrical Prosthesis With an SSVEP-Based BCI", IEEE Transactions on Biomedical Engineering Vol. 55, No.1, pp. 361-364, 1-4 February 2008.

[22] E. Hortal, D. Planelles, A. Costa, E. Ianez, A. Ubeda, J.M Azorin, and E. Fernandez, "SVM-based Brain-Machine Interface for controlling a robot arm through four mental tasks", Neurocomputing, Vol. 151, part 1, pp. 116-121, January 2014.

[23] Website: https://www.youtube.com/watch?v=zvjwaTDsEVQ 